\title{
Thermal Barrier for High-Voltage Permanent Magnet Synchronous Motor with Air-cooling Hybrid Ventilation Systems
}

\author{
$1^{\text {st }}$ Xiaochen Zhang \\ Dept. Electrical and Electronic \\ Engineering \\ University of Nottingham \\ Nottingham, UK \\ xiaochen.zhang@nottingham.ac.uk \\ $4^{\text {th }}$ Chris Gerada \\ Dept. Electrical and Electronic \\ Engineering \\ University of Nottingham \\ Nottingham, UK \\ chris.gerada@nottingham.ac.uk
}

\author{
$2^{\text {nd }} \mathrm{He}$ Zhang \\ Dept. Electrical and Electronic \\ Engineering \\ University of Nottingham Ningbo China \\ Ningbo, China \\ he.zhang@nottingham.edu.cn \\ $5^{\text {th }}$ David Gerada \\ Dept. Electrical and Electronic \\ Engineering \\ University of Nottingham \\ Nottingham, UK \\ david.gerada@nottingham.ac.uk
}

\author{
$3^{\text {rd }}$ Weili Li \\ School of Electrical and Electronic \\ Engineering \\ Beijing Jiaotong University, \\ Beijing, China \\ wlli@bjtu.edu.cn \\ $6^{\text {th }}$ Jing Li \\ Dept. Electrical and Electronic \\ Engineering \\ University of Nottingham Ningbo China \\ Ningbo, China \\ jing.li@nottingham.edu.cn
}

\begin{abstract}
This paper addresses the thermal network improvement in a $315 \mathrm{~kW}$ high-voltage solid rotor permanent magnet synchronous motor, with a hybrid air-cooling system, by the proposed thermal barrier. The multi-coupled fields, as electromagnetic fields, fluid field, and thermal fields of the machine are investigated via the finite-element analyzing. Looking at the reduction of magnet working temperatures, a thermal barrier approach is proposed to re-regular the heat transmission network, so as to control the temperature rise in magnets. The variations of loss and temperature distributions in machine affected by the thermal barrier are studied. The obtained results, be verified by the test date, shows that the thermal barrier has of effectiveness in reducing magnet working temperature, which is up to $15^{\circ} \mathrm{C}$ in case study.
\end{abstract}

Keywords—thermal barrier, PMSM, solid rotor, magnet, multi-coupled fields

\section{INTRODUCTION}

High voltage permanent magnet synchronous motor with solid rotor, has advantages of excellent performance in both starting and normal operating cases, be benefited from the solid rotor core, and have been widely used in industrial area like water treatment, well and rock, and air compressors [1].

The solid rotor makes the structure of rotor become more simple but with higher stability, which brings wide and broad potential applications in industrial field [2-5]. A lot of researches on the starting and running performance of permanent magnet synchronous motor have been perofrmed. David G proposed the design arts and analyses methods for Permanent Magnetic Synchronous Machine(PMSM), and also various design considerations like slot shapes, magnetizing patterns of controlled-PM and skewing [2]. A numerical method based on the 3-dimensional equivalent magnetic circuit network (3-D EMCN) was used to analyze a permanent magnet linear machine which has of Magnet Arrangement in [3]. Whilst some other researchers focus on the starting performance of this kind of machine[1][4].

However, for this kind of machine, the eddy current in solid rotor also results a higher working temperature, especially for the magnets with high thermal sensitivity. Currently, most of researches are looking at electromagnetic issues as topology design and output performance investigation, but rarely about the thermal management. It is necessary to investigate the thermal performance of the motor, to find a better solution which can considering both machine output features and thermal reliability well. Facing theses requests, therefore some researches have been performed in the view of thermal management[6-9]. The thermal network method has been studied in [6]. The finite element analysis (FEA) is used to solve the electromagnetic-thermal problem of the motor [8].

Considering that the eddy loss in magnets is comparatively less than those in rotor, the heat flux probably transferred from rotor core to magnet, and increase its working temperature. In this paper, a novel thermal barrier approach, which surrounds magnets in rotor slots, is proposed to reduce magnets working temperature, and whilst improve machine robustness. The thermal barrier approach has be performed on a $315 \mathrm{~kW}, 6 \mathrm{kV}$, 6 pole HVSRPM, via the electromagnetic-thermal-fluid coupled analyses. Its effectiveness could be observed from the simulated data, and also be verified by the practical experimental results.

\section{ELECTROMAGNETIC INVESTIGATION MODELING}

In this paper the case study is performed a medium power high voltage PM machine, in which the stator has of a similar geometry with those in induction machines, but with a solid iron rotor and internal insert magnets. The machine has of a rated voltage of $6 \mathrm{kV}$ and a power output of $315 \mathrm{~kW}$. Its working speed is $1000 \mathrm{rpm}$. The outer diameter of stator core is $670 \mathrm{~mm}$ and the core length is $550 \mathrm{~mm}$. The rotor has 6 magnetic poles, of each it is composed of 30 segments in axial direction. The basic structure of the machine, in the view of cross section, is shown in Fig.1, which is also the electromagnetic analysis model.

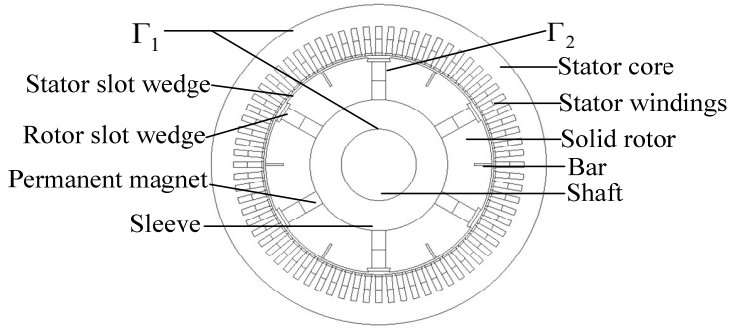

Fig. 1. Machine structure and electromanetic analysis modle. 
To perform the electromagnetic analyses more effectively, the following assumptions have been made for simplification in the solution procedure, similar to those in [9]:

1) For the operating frequency is only $50 \mathrm{~Hz}$, both the displacement current and the skin effect in coils are ignored.

2) Materials are anisotropy.

3) The material characteristics, as conductivity and permeability, are considered at the operating temperature, but ignore the thermal effects.

Based on the literature [10], the 2-D transient electromagnetic field calculation equation, the mathematical model is established, and the calculation region is determined just as shown in Fig.1.

By using the time-step finite element analyses (FEA), the time varying flux distribution inside machine is obtained, based on which the armature phase current, armature copper loss, and stator core loss are determined. During the calculation, the eddy current losses in solid conductive components, as solid rotor core, copper wedges, cage bars and permanent magnets, caused by the flux harmonics in the airgap could be calculated by (1). Within a time cycle, the total eddy loss in rotor could be [10]

$$
P_{e}=\frac{1}{\mathrm{Te}} \int_{\mathrm{Te}} \sum_{i=1}^{k} J_{e}^{2} \Delta_{e} \sigma_{r}^{-1} l_{t} \mathrm{~d} t,
$$

where, $P_{e}$ is rotor eddy current (in $\mathrm{W}$ ), $J_{e}$ is element eddy current density (in $\mathrm{A} / \mathrm{m}^{2}$ ), $\Delta_{e}$ is element area (in $\mathrm{m}^{2}$ ), $l_{t}$ is solid rotor core length (in $\mathrm{m}$ ), $\sigma_{r}$ is rotor sleeve conductivity (in $\mathrm{S} / \mathrm{m}$ ).

TABLE I. LOSSES IN SOLID ROTOR PMSM WHILE RATED LOAD OPERATING (IN W)

\begin{tabular}{|c|c|c|c|c|}
\hline \multirow{2}{*}{ Copper loss } & \multirow{2}{*}{ Iron loss } & \multicolumn{3}{|c|}{ Eddy current loss } \\
\cline { 3 - 5 } & & $\begin{array}{c}\text { Solid } \\
\text { rotor }\end{array}$ & $\begin{array}{c}\text { Copper } \\
\text { wedge }\end{array}$ & $\begin{array}{c}\text { Copper bars } \\
\text { in rotor cage }\end{array}$ \\
\hline 4389 & 5412 & 2198 & 2724 & 454 \\
\hline
\end{tabular}

\section{FLUID-THERMAL COUPLING ANALYSIS}

\section{A. Modeling for Coupling Analysis}

The solid rotor PMSM has of a hybrid air-cooling system including an inner loop and an outer loop. In the ventilation outer loop, the outside air, forced by the action of centrifugal fan, enters into the aluminum tubes to realize the heat exchange with the hot air from inner loop, and finally flows out from the outer loop air outlet, just as shown in Fig. 2.

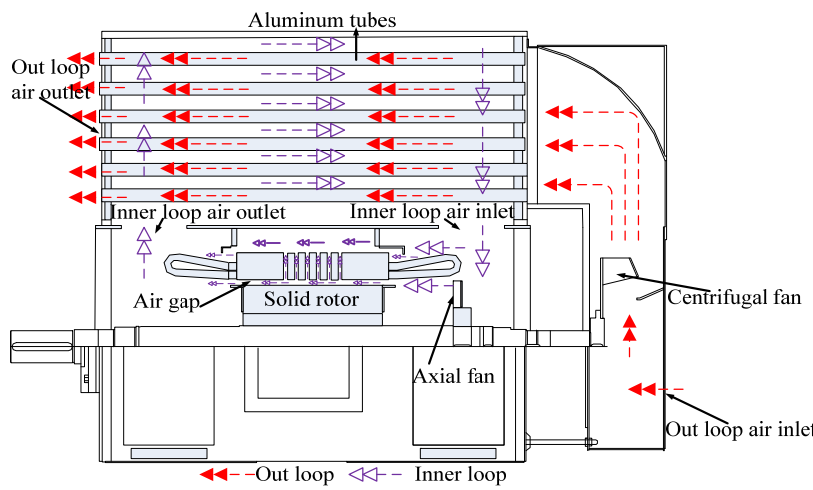

Fig. 2. Ventilating Structure of HVSR-PMSM
The cold air inside the inner loop, forced by the pressure generated from the axial fan, goes into the motor through the inlet, and passes the air-gap and stator axial ventilation ducts through the axial fan. Under the centrifugal effects caused by the rotating rotor, the cold air flows into stator radial ventilation ducts along with the air-gap, and then passes the back iron of the stator core and the stator back cooling grooves. Eventually it runs to the outlet. Then heated air enters into the cooler through machine outlet, and back into the motor inlet after be cooled, and so the cycle repeats.

The motor has asymmetrical axial and radial ventilation structures, and the flow of the air in the motor is very complicated especially with the cooler has of heat exchange tubes, so it is difficult to build the whole fluid-thermal coupling model.

Therefore, the motor internal axial fan load working point is determined by the air-flow equivalent network, in this investigation, and then the temperature field of the PMSM is calculated by the fluid-thermal coupling through the motor model and cooler model, separately. The calculation model is shown in Fig.3.

\section{B. Mathematical models}

The air flow and heat transfer in HVSR-PMSM satisfy the laws of the mass conservation, the momentum conservation, and the energy conservation. The mathematical model for fluid-thermal coupling analyzing is deduced from the control equations, just as shown in (2).

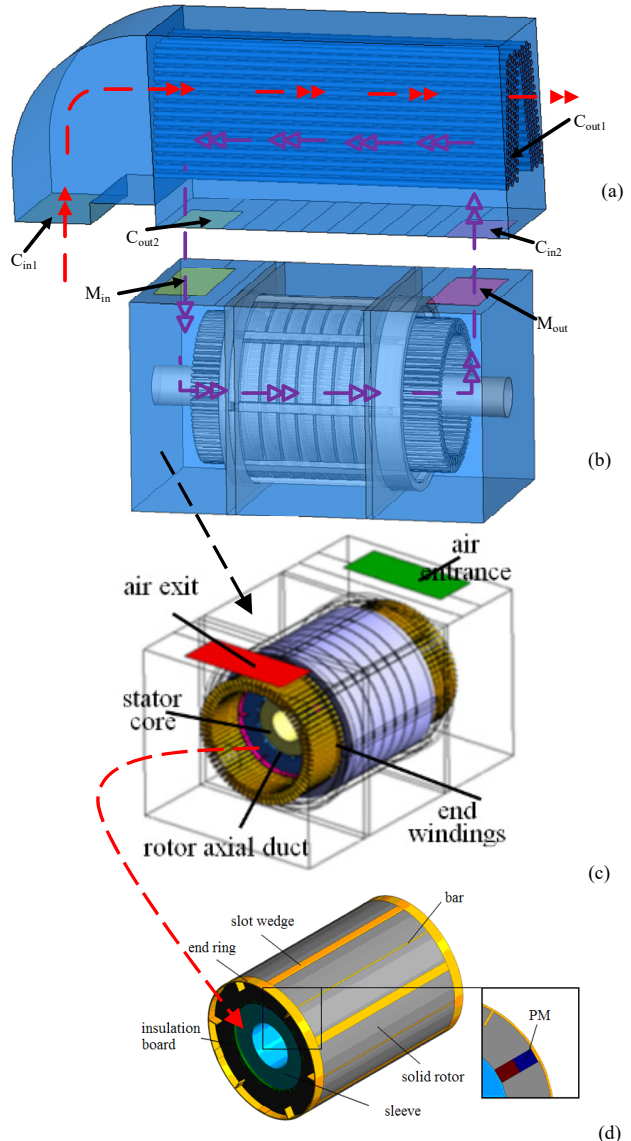

(d)

Fig. 3. Physical model for three dimensional fluid-thermal coupled analyses:(a) Cooler, (b) Machine, (c) Stator, (d) Rotor. Cin1-Out loop air inlet, Cout1-Out loop air outlet, Cout2, Min-Inner loop air inlet, Cin2, MoutInner loop air outlet. 


$$
\left\{\begin{array}{l}
\operatorname{div}(\rho \boldsymbol{u})=0 \\
\operatorname{div}(\rho \boldsymbol{u} u)=\operatorname{div}(\mu \operatorname{grad} u)-\frac{\partial P}{\partial x}+G_{u} \\
\operatorname{div}(\rho \boldsymbol{u} v)=\operatorname{div}(\mu \operatorname{grad} v)-\frac{\partial P}{\partial y}+G_{v} \\
\operatorname{div}(\rho \boldsymbol{u} w)=\operatorname{div}(\mu \operatorname{grad} w)-\frac{\partial P}{\partial z}+G_{w} \\
\operatorname{div}(\rho \boldsymbol{u} T)=\operatorname{div}\left(\frac{\lambda}{c} \operatorname{grad} T\right)+H_{T}
\end{array}\right.
$$

where, $\rho$ is the density of cooling air (in $\mathrm{kg} / \mathrm{m}^{3}$ ), $\boldsymbol{u}$ is the velocity vector, $u, v, w$ are the velocity vector in $\mathrm{x}, \mathrm{y}, \mathrm{z}$, direction of the component (in $\mathrm{m} / \mathrm{s}$ ), $P$ is fluid pressure (in $\mathrm{Pa}$ ), $\mathbf{S}_{\boldsymbol{u}}, \mathbf{S}_{\boldsymbol{v}}, \mathbf{S}_{\boldsymbol{w}}$ are the conservation of momentum of the generalized source term in all directions, $\boldsymbol{S}_{\boldsymbol{T}}$ is the heat source for energy equation, $\varnothing$ is the dynamic viscosity coefficient [in $\mathrm{kg} /(\mathrm{m} \cdot \mathrm{s})], \lambda$ is the thermal conductivity $\left[\mathrm{in} \mathrm{W} /\left(\mathrm{m} \cdot{ }^{\circ} \mathrm{C}\right)\right], c$ is the specific heat $\left[\right.$ in $\left.\mathrm{J} /\left(\mathrm{kg} \cdot{ }^{\circ} \mathrm{C}\right)\right], T$ is the temperature (in ${ }^{\circ} \mathrm{C}$ ).

From the brief analysis, the cooling air is flowing in the status of turbulent state, and the study on air flow condition in motor adopts standard $k-\varepsilon$ turbulence model [10].

$$
\left\{\begin{array}{l}
\frac{\partial\left(\rho k u_{i}\right)}{\partial x_{i}}=\frac{\partial}{\partial x_{j}}\left[\left(\mu+\frac{\mu_{t}}{\sigma_{k}}\right) \frac{\partial k}{\partial x_{j}}\right]+G_{k}-\rho \varepsilon \\
\frac{\partial\left(\rho k u_{i}\right)}{\partial x_{i}}=\frac{\partial}{\partial x_{j}}\left[\left(\mu+\frac{\mu_{t}}{\sigma_{\varepsilon}}\right) \frac{\partial \varepsilon}{\partial x_{j}}\right]+G_{1 \varepsilon} \frac{\varepsilon}{k} G_{k}-G_{2 \varepsilon} \rho \frac{\varepsilon^{2}}{k}
\end{array}\right.
$$

where, $k$ is the turbulent kinetic energy, $\varepsilon$ is the diffusion factor, $G_{k}$ is the turbulence dissipation rate, $G_{1 \varepsilon}, G_{2 \varepsilon}$ are constant, $\sigma_{\varepsilon}$ and $\sigma_{k}$ are the Planck constant.

\section{Basic hypothesis and Physical model}

According to the fluid dynamic and heat-transfer theories, some assumptions are proposed to simplify the investigation, based on geometry of the motor, which are:

1) The buoyancy effect on the cooling air flowing in ventilation ignored,

2) The velocity of air flowing in the hydride ventilation system is much slower than that of the sound velocity, and the cooling air is considered as the incompressible fluid,

3) In this investigation, only the steady equilibrium states both of fluid and heat transfer are considered, and in the control equations quantity does not change with time,

Based on the assumptions and the mathematical model above, a three-dimensional fluid-thermal coupling calculation model is determined. As shown in Fig.3, the fluid-thermal coupled calculation model, in which the air in cooler and motor are the fluid and the motor belong to the solid model.

\section{Boundary conditions}

In this study, the temperature of air in outer loop is more easier to be measured than those of air inside machine. From the experimental research, both the inlet $\left(\mathrm{C}_{\mathrm{in} 1}\right)$ and the outlet $\left(\mathrm{C}_{\text {out } 1}\right)$ temperature of air in outer loop are tested, which are list in TABLE II.

However, for the calculation boundary of machine, the inner loop inlet cooling air temperature $\left(\mathrm{M}_{\mathrm{in}}, \mathrm{C}_{\mathrm{out} 2}\right)$ is difficult to obtain from measurements. Here, the temperature iteration approach is proposed, by using which to get a more reasonable thermal boundary.
TABLE II. MEASURED VALUES OF WIND VELOCITY AND WIND TEMPERATURE OF COOLER UNDER RATED LOAD CONDITION

\begin{tabular}{|c|c|c|}
\hline Cooler & Wind velocity $(\mathbf{m} / \mathbf{s})$ & Wind temperature $\left({ }^{\circ} \mathbf{C}\right)$ \\
\hline Inlet $\mathrm{C}_{\text {in } 1}$ & 8.7 & 20.1 \\
\hline Outlet $\mathrm{C}_{\text {out } 1}$ & 9.5 & 28.3 \\
\hline
\end{tabular}

Assuming that the initial inlet temperature of the inner loop air $\left(\mathrm{M}_{\mathrm{in}}, \mathrm{C}_{\mathrm{out} 2}\right)$ is $\mathrm{T}_{\mathrm{in} 1}$, the temperature $\mathrm{T}_{\text {out1 }}$ of the inner loop air outlet $\left(\mathrm{M}_{\text {out }}, \mathrm{C}_{\mathrm{in} 2}\right)$ could be obtained by solving the fluid-thermal coupled calculation, as shown in Fig. 4(b). Then, a new temperature $T_{\text {in2 }}$ of the inner loop air inlet $\left(\mathrm{M}_{\text {in }}, \mathrm{C}_{\text {out } 2}\right)$ is obtained by solving the fluid-thermal coupling calculation model of cooler. Finally, the inner loop air inlet temperature $\mathrm{T}_{\mathrm{in} 2}$ and $\mathrm{T}_{\mathrm{in} 1}$ are compared, and the calculation will be performed repeat again, until the temperature difference $T_{d}$ less than the error allowable value $\varepsilon$. Temperature iteration calculation flow is shown in Fig.5, and the temperature judgment could be written as

$$
T_{d}=\frac{\left|T_{i n 2}-T_{i n 1}\right|}{T_{i n 1}}<\varepsilon
$$

From the inlet and outlet air temperature iteration, it indicates that the $T_{d}$ is varying obvious during the iteration process. The initial temperature (Min, Cout2) of the inner loop air inlet is $50^{\circ} \mathrm{C}$. After six iterations, the temperature (Min, Cout2) of the inner loop air inlet is $68^{\circ} \mathrm{C}$, and the iterative error is $0.47 \%$.

According to the machine geometry features, as well as of those of ventilation system, the boundary conditions foe this investigation are considered as:

1) The inlet $\left(M_{i n}\right)$ boundary conditions for a given mass flow of the motor, whose mass flow rate is $0.4 \mathrm{~kg} / \mathrm{s}$, and the inlet temperature is $68^{\circ} \mathrm{C}$.

2) The inlet $\left(\mathrm{C}_{\mathrm{in} 1}\right)$ boundary conditions for a given velocity inlet of the cooler, which has a velocity magnitude of $8.7 \mathrm{~m} / \mathrm{s}$, and a temperature of $20.1^{\circ} \mathrm{C}$.

3) The inlet $\left(\mathrm{C}_{\mathrm{in} 2}\right)$ boundary conditions for a given mass flow of the cooler, whose mass flow rate is $0.4 \mathrm{~kg} / \mathrm{s}$. The inlet temperature is $91.7^{\circ} \mathrm{C}$.

4) The outlet $\left(\mathrm{M}_{\text {out }}, \mathrm{C}_{\text {out } 1}, \mathrm{C}_{\text {out2 }}\right)$ boundary conditions is a given pressure of the motor and the cooler, of which the ambient pressure is $1 \mathrm{~atm}$.

\section{E. Results and Analyses}

From the fluid-thermal coupled calculation, the fluid flowing statues and the temperature distribution in machine are obtained. In the studied solid rotor PMSM, there are radial ventilation ducts in stator core, which have been placed in 6 axial locations and there 12 ducts in circumstance at each location. From the rotor axial fan end to the shaft end, those axial ducts are numbered as No.1 to No.6. The No.1, No.2 and No.3 stator radial ventilation ducts belong to the out-wind zone, and the No.4, No.5 and No.6 stator radial ventilation duct belong to in-wind zone.

As shown in Fig. 4, the fluid velocity distribution in one stator radial ducts cross section, which varies obvious. The maximum flow velocity of air in No.1 duct is $1.69 \mathrm{~m} / \mathrm{s}$ and such flowing velocity declines to $1.2 \mathrm{~m} / \mathrm{s}$ at the stator tooth root, and reach the minimum at the yoke back. The maximum flow velocity of air in the No.2 duct is $1.3 \mathrm{~m} / \mathrm{s}$, but it is only $0.6 \mathrm{~m} / \mathrm{s}$ for fluid in the No.3 duct. 


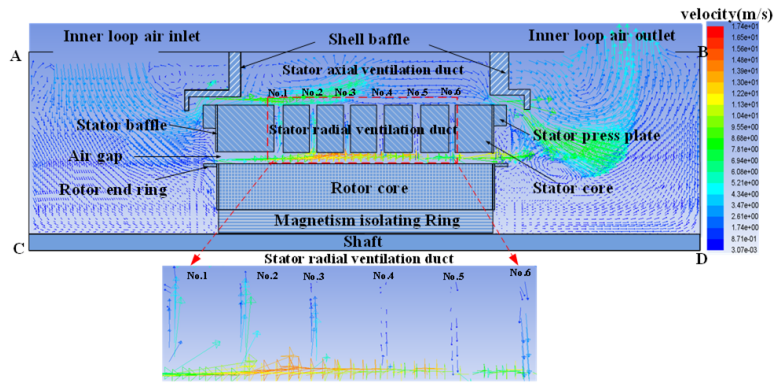

Fig. 4. Fluid distribution in machine cross section.

Fig. 5 shows the temperature distributions of fluid in stator radial ducts, in which the region $\mathrm{A}$ is the temperature of fluid closes to entrance at the air-gap side, and region B is that near the stator yoke back outlet. It can be seen that, temperature variation of cooling air along the radial direction are comparatively larger than that in the tooth and yoke region.

Of the air in the No.1 duct, the temperature at entrance at the air-gap side is $113^{\circ} \mathrm{C}$ and it reaches to $120^{\circ} \mathrm{C}$ at the end of region A. Due to the velocity change of tooth is very small, the air temperature is stable at $118^{\circ} \mathrm{C}$ in region of stator tooth. The air temperature rises about 2 to $4^{\circ} \mathrm{C}$ during the yoke region. Whilst in area $\mathrm{B}$, the fluid temperature reaches the highest about $124^{\circ} \mathrm{C}$, and then it fell sharply.

Temperature of the stator winding along the axial direction is increasing firstly, and then it begins to decrease. The highest temperature appears at the axial position between the No. 4 and No. 5 radial ducts, which is about $150.9^{\circ} \mathrm{C}$

As shown in Fig. 6, rotor temperature increases obvious from the fan end to the shaft end along with the axial direction, which is up to $40^{\circ} \mathrm{C}$. After the insertion of thermal barrier, made of high thermal resistance material as epoxy resin, more heat generated in rotor core be transferred to stator. The working temperature of magnet reduce up to $15^{\circ} \mathrm{C}$, and whilst rotor core temperature increases $0.6^{\circ} \mathrm{C}$, as shown in Fig. 6 b).

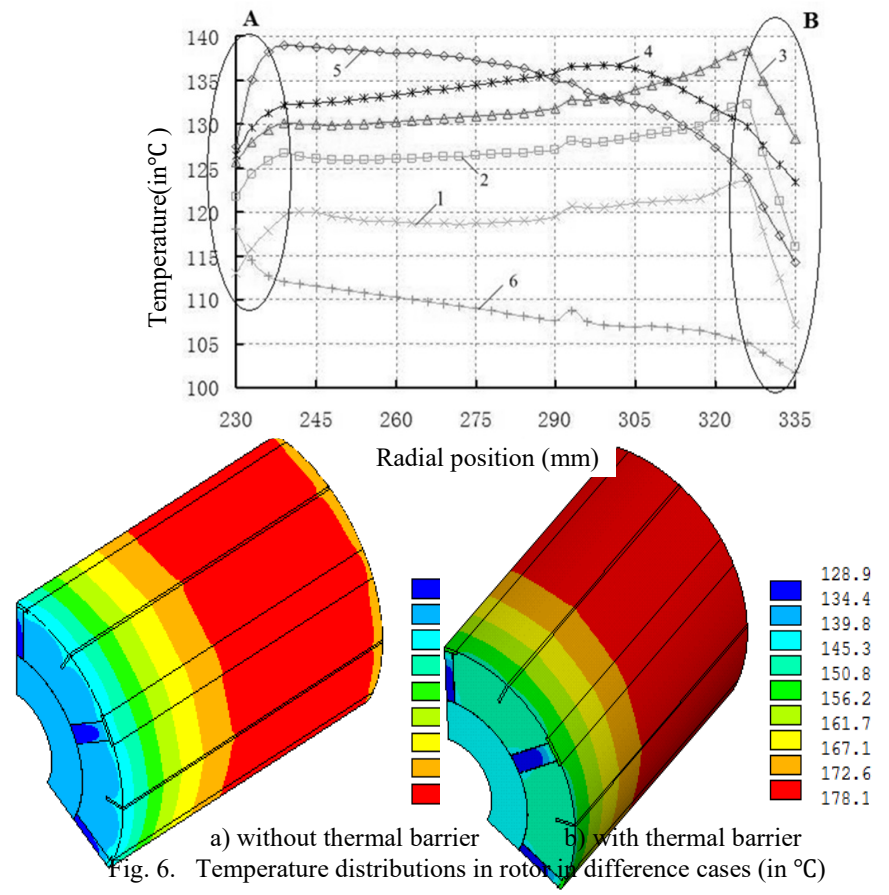

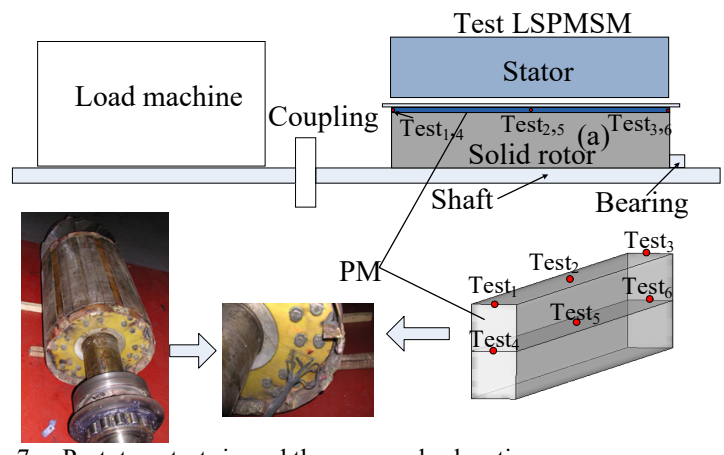

Fig. 7. Prototype test rig and thermocouples locations.

For experimental investigation, five NTC thermocouples are fixed in prototype rotor, and the comparison of the measured and the calculated date at test positions are list in Table III. The maximum calculated error is less than $8 \%$, and it is basically in accordance with the actual requirements.

TABLE III. The main parameters of the MOTOR $\left(\mathrm{IN}^{\circ} \mathrm{C}\right)$

\begin{tabular}{|c|c|c|c|}
\hline Points & Measured & Calculated & Error (\%) \\
\hline 1 & 142.7 & 154.4 & +7.57 \\
\hline 2 & 144.7 & 152.4 & +5.05 \\
\hline 3 & 169.5 & 170.6 & +0.68 \\
\hline 4 & 176.5 & 169.6 & -4.15 \\
\hline 5 & 181.4 & 176.5 & -2.79 \\
\hline
\end{tabular}

\section{CONCLUSION}

For solid rotor PMSM, the eddy loss in rotor core becomes obvious large, and makes more heat transferred from rotor core to magnets. A thermal barrier is proposed to prevent such heat flow. Via the case studying on a $315 \mathrm{~kW}$ solid rotor HPMSM, the magnet working temperature could reduce about $13 \%$. The obtained conclusion could provide a reference for the thermal network design of solid rotor machines.

\section{REFERENCES}

[1] K. Kurihara, M.A. Rahman. "High-efficiency line-start interior permanent-magnet synchronous motors", IEEE Trans. Ind. Appl., vol. 40, no. 3, pp. 789-796. May.2004

[2] D. Gerada, A. Mebarki, N. L. Brown, C. Gerada, et.al, "High-Speed Electrical Machines: Technologies, Trends, and Developments," IEEE Trans. Ind. Electron., vol. 61, no. 6, pp. 2946-2959, Jun. 2014.

[3] X. Zhang, W. Li, B. Kou, et al. "Electro-thermal combined optimization on notch in air cooled High Speed Permanent Magnetic Generator," IEEE Trans. Mags., vol. 51, no. 1, \# 8200210, Jun. 2014.

[4] ZB. Cao, WL. Li, JY. Li, XC. Zhang, D. Li , MW. Zhang. "Research on the Temperature Field of HVHP-LSPM with Different Rotor Cage Structure". Energies 2017, vol. 10, no. 11, \#1829

[5] J. Lee, H.W. Lee, "The Performance Prediction of Controlled-PM LSM in Various Design Schemes by FEM". IEEE Trans. Magn., vol. 36, no. 4, pp. 1902-1905, Jul. 2000.

[6] N. Bracikowski, M. Hecquet, P. Brochet, S.V. Shirinskii, "Multiphysics Modeling of a Permanent Magnet Synchronous Machine by Using Lumped Models". IEEE Trans. Ind. Electron, vol. 59, no. 6, pp. 2426-2437, Jun. 2012,

[7] X. Zhang, W. Li, C. Gerada, H. Zhang, et.al. "CQICO and multiobjective thermal optimization for high speed PM generator," IEEE Trans. Mags, vol. 53, no. 6, 8201604, Jun. 2017.

[8] W. Li, X. Zhang, S. Cheng, "Study of solid rotor line-start PMSM operating". Proc. Intel. Conf. Electrical Machines, pp. 1803-1809, 1720, Oct 2008 Wuhan, China.

[9] W. Li, H. Qiu. "Influence of Copper Plating on Electromagnetic and Temperature Fields in a High-Speed Permanent-Magnet Generator". IEEE Trans Magn. 2012. vol. 48, no. 2, pp. 2247-2253.

[10] W. Li, X. Zhang, S. Cheng, and J. Cao, "Thermal Optimization for a HSPMG Used for Distributed Generation Systems," IEEE Trans. Ind. Electron., vol. 60, no. 2, pp. 474-482, Feb. 2013. 